\title{
Implicações da pouca preocupação e percepção familiar no sobrepeso infantil no município de Curitiba, PR, Brasil
}

\author{
The implications of family lack of concern and perception of \\ obesity in childhood in the city of Curitiba, State of Paraná, Brazil
}

Daniele Ferreira Barbosa dos Santos ${ }^{1}$

Giovanna Chipon Strapasson ${ }^{1}$

Samantha Daliana Pacheco Golin ${ }^{1}$

Eliane Carneiro Gomes ${ }^{2}$

Grace Maria Ferreira de Castro Wille ${ }^{2}$

Sandra Mara Woranovicz Barreira ${ }^{2}$

${ }^{1}$ Programa de PósGraduação em Ciências Farmacêuticas, Universidade Federal do Paraná (UFPR). Av. Prefeito Lothario Meissner, Jardim Botânico. 80210-170 Curitiba PR Brasil.dani_iep@msn.com ${ }^{2}$ Departamento de Farmácia, Setor de Ciências da Saúde, UFPR. Curitiba PR Brasil.

\begin{abstract}
The scope of this study was to relate child obesity with the family's perception of the child's silhouette's and concern with excess weight in childhood A cross-sectional study was conducted among children aged between 20 and 48 months who frequent Health Units or are preschoolers in the city of Curitiba from June to December 2014. A questionnaire was used to collect data relating to family income, weight, height, perception of child silhouette's and assess concern about obesity by parents. Overweight and obesity was not correctly perceived by relations (kap$p a=0,11)$ and the vast majority of parents did not know the child's height and weight and were not even concerned with childhood obesity. In the population studied, children from lower income family were more likely to suffer from overweight in childhood $(p=0,02)$. It is necessary to invest in the sensitization of families, since it is extremely important to pay attention to obesity from childhood onwards, thus preventing new cases and avoiding problems in adult life.
\end{abstract}

Key words Childhood obesity, Family perception, Socioeconomic level
Resumo O presente trabalho propôs relacionar o sobrepeso infantil com a percepção familiar da silhueta da criança e a preocupação com o excesso de peso na infância. Foi realizado um estudo transversal com crianças de 20 a 48 meses de idade usuárias de Unidades de Saúde ou centros de ensino infantil de Curitiba, no período de junho a dezembro de 2014. Fez-se uso de questionário para coletar dados referentes a renda familiar, peso, altura, percepção da silhueta da criança e verificar a preocupação com o excesso de peso. O sobrepeso e a obesidade não foram percebidos corretamente pelos responsáveis $(k a p p a=0,11)$ e grande parte destes não sabiam o peso e altura da crianças e nem estavam preocupados com excesso de peso. $\mathrm{Na}$ população estudada, as crianças de famílias de menor renda apresentaram mais chance de desenvolver sobrepeso na infância $(p=0,02)$. Se faz necessário investir na sensibilização das famílias, pois é de extrema importância a atenção à obesidade desde a infância prevenindo assim novos casos e evitando agravos na vida adulta.

Palavras-chave Obesidade infantil, Percepção familiar, Nível socioeconômico 


\section{Introdução}

O excesso de peso expressa o desequilíbrio entre ingestão e utilização de calorias pelo organismo humano e se reflete no aumento do peso corpóreo pelo aumento da massa adiposa, o que pode levar a agravos de saúde ${ }^{1}$. A obesidade infantil atualmente se caracteriza como uma epidemia, sendo um grande desafio à saúde pública. Seu combate pode evitar outras doenças crônicas não transmissíveis como os acidentes cardiovasculares isquêmicos e o diabetes na vida adulta ${ }^{2-4}$.

Segundo o SISVAN-Curitiba ${ }^{5}$, entre os curitibanos menores de 4 anos, as prevalências de muito baixo peso, baixo peso e peso elevado para a idade, em 2012, foram de 1,33\%; 3,33\% e 4,08 respectivamente. A prevalência do peso elevado tem superado a do baixo peso desde 1996 nesta população.

A obesidade é uma doença de causas múltiplas que envolvem fatores genéticos (metabólicos) e ambientais (nutricionais e psicossociais), e parecem interagir, levando a um balanço calórico positivo, sendo difícil estabelecer a contribuição de cada um ${ }^{4,6}$. Acredita-se que as causas exógenas respondam por $95 \%$ dos casos mundiais de obesidade, sendo as principais: a maior disponibilidade e ingestão de alimentos hiperlipídicos; a mudança do estilo de vida ativa para sedentária e o aumento das porções ${ }^{4}$.

Outros fatores em estudos atualmente são: nível socioeconômico, comportamento familiar, obesidade dos pais, privação afetiva, falta de percepção familiar do sobrepeso da criança, entre outros. Há implicações do papel da família na obesidade das crianças e, mais especificamente, do papel das mães ${ }^{4,7,8}$. O reconhecimento do excesso de peso das crianças pode ser o requisito necessário para a procura de ajuda profissional e promover uma melhora na adesão ao tratamento, mas a percepção familiar distorcida faz com que o excesso de peso seja percebido como normal e desejável, impossibilitando tal ajuda ${ }^{8-10}$.

Um estudo alemão que avaliou a percepção materna em relação à silhueta do seu filho mostrou que parte das mães de crianças mais novas preferiam que o filho fosse mais gordinho ${ }^{10}$. Outro estudo de percepção, norte-americano, apontou que parte dos pais não estava preocupada com o peso dos filhos e subestimou este dado ${ }^{11}$.

Até o presente momento, não há publicações disponíveis avaliando a influência da falta de percepção familiar sobre o perfil nutricional dos seus filhos no desenvolvimento da obesidade no Sul do Brasil. Assim o presente projeto visa re- lacionar o sobrepeso e a obesidade infantil com a percepção e preocupação familiar na cidade de Curitiba (PR).

\section{Método}

\section{Desenho e população}

Um estudo transversal foi conduzido com 514 crianças de 20 a 48 meses de idade, dos sexos masculino e feminino, que não apresentassem síndromes e que frequentassem as Unidades de Saúde (US) e centros de ensino infantil selecionados do município de Curitiba, PR. Foram selecionadas aleatoriamente 2 Unidades de Saúde de cada um dos 9 distritos sanitários do município, posteriormente a Secretaria Municipal de Educação indicou 14 centros municipais de ensino infantil e outros 11 centros particulares aceitaram participar do estudo realizado de junho a setembro de 2014.

A casuística desse estudo foi composta pela amostra do estudo de Baltazar ${ }^{12}$, que investigou a influência do aleitamento no peso das crianças curitibanas realizado em 2014, o qual teve delineamento da amostra calculada sobre os nascidos vivos de Curitiba em 2011 (25.247 crianças) considerando a prevalência de sobrepeso infantil do município de 4,08\% no ano de 2012 (pelo menos 166 crianças, com nível de confiança de $95 \%$ e erro de estimativa de $3 \%$ ).

\section{Coleta de dados e análise}

Foi elaborado um questionário para coletar dados referentes a renda familiar, peso, altura, percepção da silhueta da criança pelo responsáveis e verificar se havia preocupação, por parte da família, que a criança viesse a ficar com excesso de peso.

O questionário possuía questões abertas e fechadas e foi aplicado às famílias, escolhidas aleatoriamente dentre as usuárias de Unidades de Saúde, em forma de entrevista, pela pesquisadora ou pelas agentes comunitárias. Neste grupo, o peso e a altura das crianças eram coletados na própria Unidade de Saúde por enfermeiras, conforme as orientações para a coleta e análise de dados antropométricos em serviços de saúde do SISVAN/MS ${ }^{13}$.

Nos centros de ensino infantil (11 privadas e 14 públicas), o mesmo questionário, com instruções para o autopreenchimento, foi entregue aleatoriamente pela diretora aos pais. Posterior- 
mente, a pesquisadora ou nutricionista do centro infantil realizava as coletas antropométricas conforme as orientações do SISVAN/MS ${ }^{13}$.

Os dados antropométricos obtidos foram digitados no software Anthro da WHO e para a avaliação do excesso de peso das crianças, foi utilizado o indicador IMC para idade (IMC/I) expresso em escore z. O excesso de peso foi definido como valor de IMC/I superior ao escore z+2. A curva WHO 2006-2007 para avaliação do estado nutricional de crianças foi utilizada como distribuição de referência ${ }^{14}$.

Procedeu-se com análises descritivas das variáveis investigadas (sexo, faixa etária da criança, renda familiar e preocupação familiar) e para verificar a concordância dos pais quanto à percepção da silhueta atual da criança com a real situação nutricional, foi utilizado o coeficiente Kappa (k) proposto por Cohen, no qual quanto mais próximo de 1 maior a concordância entre a percepção e a realidade. Considerou-se $\mathrm{k}<0,4$ como concordância fraca e $\mathrm{k}>0,8$ como concordância forte.

Para verificar marginalmente a associação entre as variáveis de sexo, faixa etária da criança, renda familiar, preocupação familiar com o excesso de peso dos filhos, com o IMC da criança foi usado o Teste do Qui-quadrado - o qual determinou a diferença de proporção nos resultados obtidos do grupo com sobrepeso e do grupo sem sobrepeso. Quando os pressupostos desse teste não foram atendidos utilizou-se o Teste Exato de Fischer. Nesta análise, o grupo sobrepeso incluiu crianças em estado de vigilância para o sobrepeso, com sobrepeso e obesas; as demais crianças eram do grupo sem sobrepeso. Para estes testes foi utilizado o pacote estatístico R Core Team 2014, A language and environment for statistical computing ${ }^{15}$.

\section{Aspectos éticos}

O estudo foi aprovado pelos Comitês de Ética em Pesquisa do Setor de Ciências da Saúde da Universidade Federal do Paraná, da Secretaria Municipal da Saúde e da Secretaria Municipal de Educação. O Termo de Consentimento Livre e Esclarecido foi apresentado ou encaminhado juntamente com os formulários da pesquisa.

\section{Resultados e discussão}

Aceitaram participar do estudo 514 famílias, doravante nomeada "amostra T". Dos 514 questio- nários respondidos, somente 297 responsáveis $(57,78 \%)$ sabiam dizer qual o peso e a altura da criança, 25 (4,86\%) tinham dados biologicamente implausíveis, 192 (37,35\%) não souberam responder ou sabiam somente o peso, portanto, as análises descritivas e estatísticas foram realizadas com a amostra parcial, doravante nomeada “amostra P" (297 crianças). Para não perder mais formulários, as demais variáveis investigadas quando não continham resposta foram consideradas como "Não responderam".

Os questionários foram respondidos pelas mães em sua grande maioria $(263 ; 88,55 \%), 14$ $(4,71 \%)$ por avós, 17 (5,72\%) pelos pais da criança, $2(0,67 \%)$ por tias e um (0,34\%) pela irmã. O grupo das crianças em estudo era constituído por 128 meninas e 169 meninos, 43,10\% e 56,9\% respectivamente. A amostra teve $60,61 \%$ de crianças de 25 a 36 meses de idade.

As curvas geradas no software Anthro, que podem ser visualizadas no Gráfico 1, mostram que a grande maioria da amostra $\mathrm{P}$ se encontrava com peso e altura adequados, no entanto deslocada à direita com tendência ao sobrepeso quando comparada a curva normal da WHO de IMC para idade e, ainda, a amostra era mais baixa quando comparada a curva normal de altura para idade da WHO.

Os perfis nutricionais encontrados foram: 5 (1,68\%) casos com baixo peso, 24 (8,08\%) em estado de vigilância para baixo peso, 153 (51,52\%) eutróficos, $79(26,60 \%)$ em vigilância para sobrepeso, $35(11,78 \%)$ estavam com sobrepeso e $1(0,34 \%)$ com obesidade. Comparando as crianças com baixo peso e as com sobrepeso e obesa nota-se uma tendência para obesidade, sendo mais evidente quando somadas as crianças ainda eutróficas, porém em estado de vigilância.

A prevalência do sobrepeso e obesidade foi de 36 casos (12,12\%). Esta prevalência percentual coincide com o resultado obtido pelo trabalho de Ferreira et al. ${ }^{16}$ em um centro municipal de ensino infantil de Curitiba.

O município de Curitiba vivencia um momento em que a prevalência do excesso de peso é maior que o déficit de peso para idade. Conforme o SISVAN ${ }^{5}$, o perfil nutricional dos curitibanos menores de 4 anos, nos últimos anos, apresentou um aumento da taxa de peso elevado de 3,01\% em 2001 para 4,08\% em 2012. Os achados deste trabalho estão de acordo com os dados do SISVAN, quando se refere a maior prevalência do sobrepeso e obesidade em relação ao baixo peso.

Os casos de sobrepeso foram detectados em todos as distritos sanitários, em todas as faixas 

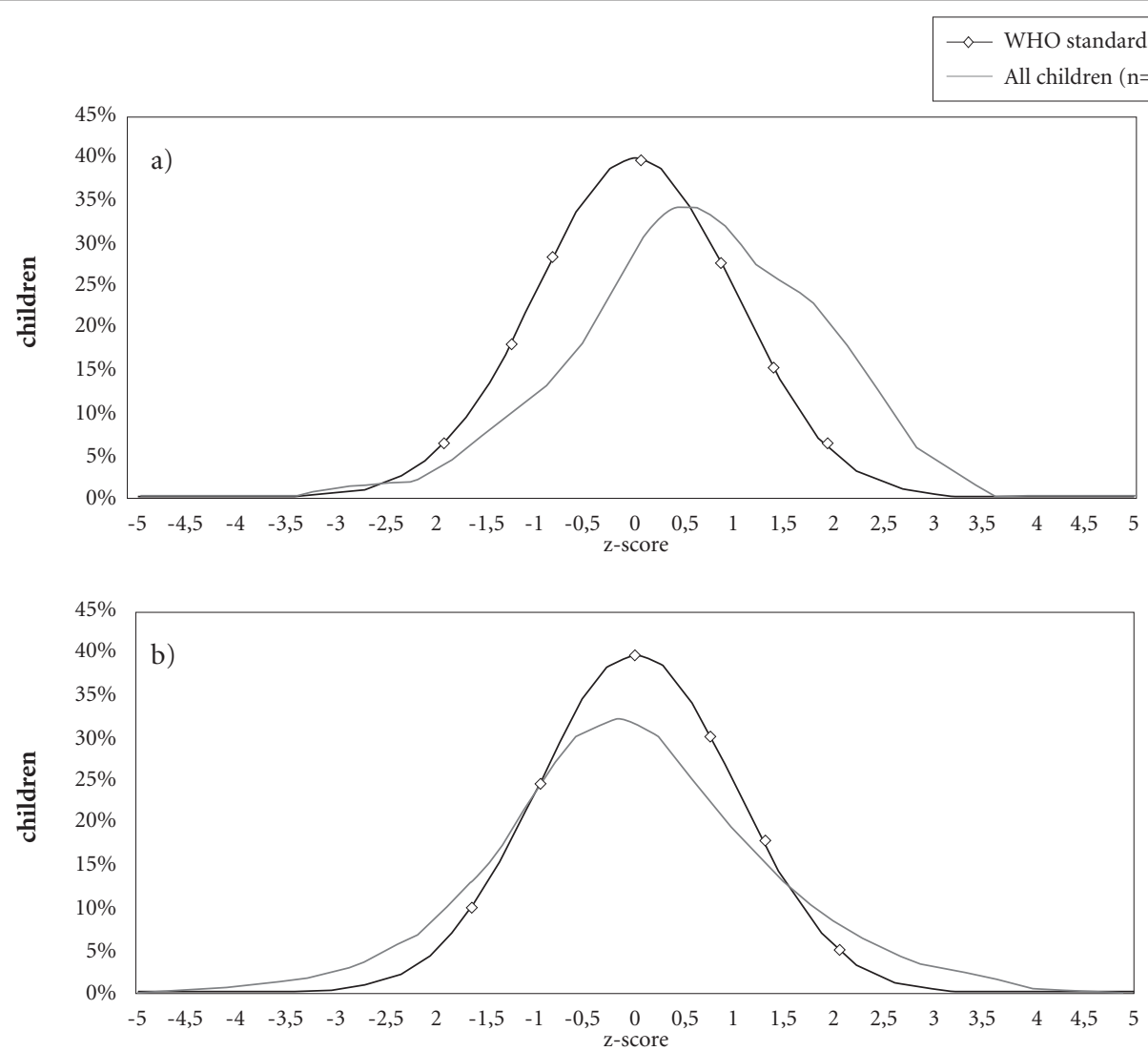

Gráfico 1. Curvas padrão da WHO e da amostra P: a) IMC-para-idade e b) altura para idade.

etárias $(\mathrm{p}=0,69)$, em ambos os sexos $(\mathrm{p}=0,8)$ e em famílias de maior e menor poder aquisitivo $(\mathrm{p}=0,02)$. Para as primeiras variáveis o teste quiquadrado demonstrou que os grupos eram homogêneos, isto é, não indicou diferença estatística significativa com nível de incerteza de $10 \%$. No entanto, as crianças que eram de famílias que ganhavam $\geq 3$ salários tinham menores chances para o sobrepeso $(\mathrm{p}=0,02)$ (Tabela 1$)$.

Sabe-se que famílias de menor nível socioeconômico procuram alimentos mais calóricos, processados, fastfood, etc ${ }^{4}$. Assim, à medida que alimentos saudáveis - peixes, carnes magras, vegetais e frutas frescas - estão menos disponíveis para indivíduos de condições mais restritas, a relação entre obesidade e menor nível socioeconômico é observada em países em desenvolvimento ${ }^{17}$.

Acerca da percepção da silhueta da criança: $28(9,43 \%)$ responsáveis consideraram que seu filho estava muito magro ou magro, 253 (85,19\%) acreditavam que estava com peso adequado, 9 $(3,03 \%)$ consideraram que estava acima do peso e $7(2,36 \%)$ questionários não continham resposta para esta questão. No Gráfico 2 foi evidenciada a distorção quando comparados os perfis nutricionais encontrados com a percepção que os responsáveis tinham da criança, tanto para as crianças magras e acima do peso, consideradas eutróficas, quanto para as que, sendo eutróficas foram consideradas magras ou com sobrepeso. Isso pode gerar uma desnutrição por falta ou por excesso. Para avaliar a concordância da percepção dos pais e a realidade quanto à silhueta atual da criança foi utilizada a estimativa kappa $(k)$, a qual foi de $\mathrm{k}=0,11$ com $\mathrm{IC}_{95 \%}(\mathrm{k})=(-0,003 ; 0,2323)$, o que confirma a fraca concordância entre a percepção dos pais e a realidade.

Quanto à preocupação de que o filho fique com sobrepeso, $135(45,45 \%)$ assinalaram estar "nada preocupada"; 61 (20,54\%) "pouco preocupada”; 53 (17,85\%) "preocupada” e $45(15,15 \%)$ 
Tabela 1. Prevalência de sobrepeso e valor de p, segundo variáveis sociodemográficas.

\begin{tabular}{|c|c|c|c|c|c|c|}
\hline \multirow[t]{2}{*}{ Variáveis } & \multirow[t]{2}{*}{ Categorias } & \multicolumn{2}{|c|}{$\begin{array}{c}\text { Baixo peso, } \\
\text { vigilância } \\
\text { para baixo } \\
\text { peso e } \\
\text { eutróficos }\end{array}$} & \multicolumn{2}{|c|}{$\begin{array}{l}\text { Vigilância } \\
\text { para } \\
\text { sobrepeso, } \\
\text { sobrepeso e } \\
\text { obesidade }\end{array}$} & \multirow[t]{2}{*}{$P$ valor } \\
\hline & & $\mathbf{n}$ & $\%$ & $\mathbf{n}$ & $\%$ & \\
\hline \multirow[t]{5}{*}{ Idade da criança } & De 20 a 24 meses & 41 & 0,55 & 33 & 0,45 & - \\
\hline & De 25 a 30 meses & 79 & 0,63 & 47 & 0,37 & - \\
\hline & De 31 a 36 meses & 33 & 0,61 & 21 & 0,39 & - \\
\hline & De 37 a 42 meses & 25 & 0,66 & 13 & 0,34 & - \\
\hline & De 43 a 48 meses & 4 & 0,8 & 1 & 0,2 & 0,69 \\
\hline \multirow[t]{2}{*}{ Sexo da criança } & Feminino & 80 & 0,62 & 48 & 0,38 & - \\
\hline & Masculino & 102 & 0,6 & 67 & 0,4 & 0,8 \\
\hline \multirow[t]{2}{*}{ Renda da família } & $<3$ salários & 47 & 0,51 & 46 & 0,49 & - \\
\hline & $\geq 3$ salários & 122 & 0,66 & 62 & 0,34 & $0,02^{\star}$ \\
\hline \multirow[t]{2}{*}{ Preocupação com o excesso de peso } & Nada ou pouco preocupados & 118 & 0,6 & 78 & 0,4 & - \\
\hline & Preocupados & 61 & 0,62 & 37 & 0,38 & 0,83 \\
\hline
\end{tabular}

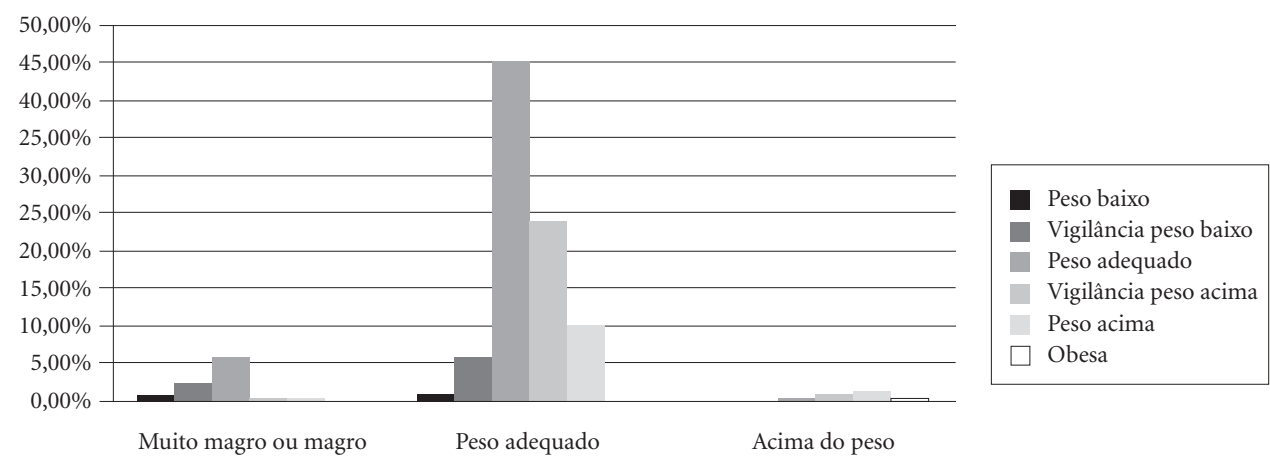

Gráfico 2. Percepção da família quanto ao perfil nutricional das crianças de acordo com o perfil nutricional real.

"muito preocupada” e apenas três $(1,01 \%)$ famílias não responderam esta pergunta.

A maioria dos responsáveis não estava preocupada com o excesso de peso das crianças, pois as consideravam com peso adequado. No entanto, ter preocupação parece não afetar o comportamento da família, tendo em vista que foram encontrados casos de sobrepeso também nas famílias preocupadas. Dos cuidadores das crianças acima do peso, $83,33 \%$ consideravam a silhueta adequada e 52,78\% estavam preocupados com o excesso de peso que os filhos poderiam desenvolver. O teste de Qui-quadrado mostrou que não existe diferença entre o grupo das famílias nada e pouco preocupadas com o grupo das famílias preocupadas na tendência para o sobrepeso ( $\mathrm{p}$ $=0,83$ ), portanto o desfecho de sobrepeso independe desta variável na população estudada.

O presente estudo mostrou que, bem como nos estudos de Warschburger e Kroller $^{10}$ e de Wright $^{11}$, grande parte dos responsáveis não acompanhavam o desenvolvimento corporal da criança $(37,35 \%$ da amostra $\mathrm{T})$, não reconheciam e não se importavam com o excesso de peso, enquanto que $21,21 \%$ dos que conheciam o peso e altura tinham uma visão distorcida do perfil nutricional dos filhos.

Evidenciou-se que é considerável a chance das crianças eutróficas mas em vigilância para sobrepeso desenvolverem o sobrepeso visto que 
as famílias deste grupo de crianças não perceberam que os filhos se encontravam no limite de eutrofia e estavam nada ou pouco preocupadas com o excesso de peso.

Durante as entrevistas, muitas falas sugeriram que os responsáveis preferem que a criança seja mais gordinha para suportar possíveis doenças, sendo maior a preocupação com a magreza. Esta questão é abordada por Camargo et al. ${ }^{8}$, como uma crença cultural materna em que o sobrepeso não é visto como um problema, já a magreza está associada com problemas de saúde e adiciona as crenças: "criança gordinha é saudável", "o crescimento irá normalizar o peso". Para Rodrigues $^{9}$, algumas mães, principalmente as de baixo nível socioeconômico, por medo de seus filhos se tornarem desnutridos, podem super alimentá-los, levando ao excesso de peso.

As limitações encontradas neste estudo foram: coletas antropométricas realizadas ora pelas enfermeiras das Unidades de Saúde ora pela pes- quisadora ou nutricionistas do centros de ensino, ausência de dados de autopercepção corporal dos responsáveis e estado nutricional dos cuidadores.

\section{Considerações finais}

O baixo nível socioeconômico foi identificado como fator de risco na população estudada o que sugere à necessidade de intensificar ações de prevenção junto a estas famílias.

A respeito do excesso de peso, é importante que exista sensibilização das famílias para a prevenção de novos casos e evitar que indivíduos com sobrepeso venham a se tornar obesos com agravos na vida adulta. Por meio de campanhas e palestras continuadas é possível alcançar a conscientização e o objetivo de prevenção nesta população. Sugere-se também capacitação às agentes comunitárias sobre o tema, pois elas são ferramentas importantíssimas de informações de saúde à população.

\section{Colaboradores}

DFB Santos trabalhou na concepção, delineamento, análise e interpretação dos dados, redação do artigo e aprovou a versão a ser publicada. GC Strapasson contribuiu para a concepção, análise e interpretação dos dados, e aprovou a versão a ser publicada. SDP Golin contribuiu para a concepção, delineamento, redação do artigo e aprovou a versão a ser publicada. EC Gomes auxiliou no delineamento, redação do artigo, sua revisão crítica e aprovou a versão a ser publicada. GMFC Wille e SMW Barreira colaboraram na concepção, delineamento, análise e interpretação dos dados, redação do artigo e aprovou a versão a ser publicada. 


\section{Agradecimentos}

As autoras agradecem aos agentes comunitários e à estudante de graduação em Farmácia, e a Isabella Borja pela colaboração na coleta dos dados. Expressam sua gratidão também às famílias que aceitaram participar do estudo e às parcerias formadas com a Secretaria Municipal de Saúde e Secretaria Municipal de Educação representadas pelos seus gestores e demais profissionais envolvidos. À Coordenação de Aperfeiçoamento de Pessoal de Nível Superior pela bolsa de mestrado concedida à autora.

\section{Referências}

1. World Health Organization (WHO). Obesity and overweight. [página da Internet] [acessado 2013 abr 15]. Disponível em: http://www.who.int/mediacentre/ factsheets/fs311/en/

2. Su C, Zhang B, Wang YF, Jia XF, Xue H, Wang HJ. Epidemics of overweight and obesity among growing childhood in China between 1997 and 2009: Impact of Family Income, Dietary Intake, and Physical Activity Dynamics. Chin Med J 2015; 128(14):1879-1886.

3. George AM, Jacob AG, Fogelfeld L. Lean diabetes mellitus: An emerging entity in the era of obesity. World $J$ Diabetes 2015; 6(4):613-20.

4. Sahoo K, Sahoo B, Choudhury AK, Sofi NY, Kumar R, Bhadoria AS. Childhood obesity: causes and consequences. J Family Med Prim Care 2015; 4(2):187-192.

5. Curitiba. Secretaria Municipal de Saúde. Sistema de Vigilância Alimentar e Nutricional. Perfil Nutricional dos Usuários das Unidades de Saúde. [acessado 2013 jun 6]. Disponível em: http://www.saude.curitiba.pr.gov. br/images/vigilancia/arquivos/epidemiologica/ indicadores/BOLETIM\%20SISVAN\%202011.pdf

6. Silventoinen K, Rokholm B, Kaprio J, Sorensen TIA. The genetic and environmental influences on childhood obesity: a systematic review of twin and adoption studies. Int J Obesity (Lond) 2010; 34(1):29-40.

7. Hoog ML, Eijsden MV, Stronks K, Gemke RJBJ, Vrijkotte TGM. Overweight at age two years in a multi-ethnic cohort (ABCD study): the role of prenatal factors, birth outcomes and posnatal factors. $\mathrm{BMCPu}$ blic Health 2011; 11:611.

8. Camargo APPM, Barros AAF, Antônio MARGM, Giglio JS. A não percepção da obesidade pode ser um obstáculo no papel das mães de cuidar de seus filhos. Cien Saude Colet 2013; 18(2):323-333.

9. Rodrigues L. Obesidade infantil. In: Accioly E, Saunders C, Lacerda EMA, organizadores. Nutrição em Obstetrícia e Pediatria. Rio de Janeiro: Guanabara Koogan, 2009. p. 369-392.

10. Warschburger P, Kroller K. Childhood overweight and obesity: maternal perceptions of the time for engaging in child weight management. BMS Public Health 2012; 12:295.

11. Wright DG. Parental perception of preschool child body weight. J Pediatr Nurs 2011; 26(5):435-445.

12. Baltazar DFBS. Fatores que influenciam o sobrepeso de crianças menores de 4 anos usuárias do SUS e centros de ensino infantil do município de Curitiba [dissertação]. Curitiba: Universidade Federal do Paraná; 2015.

13. Brasil. Ministério da Saúde (MS). Orientações para a coleta e análise de dados antropométricos em serviços de saúde: Norma Técnica do Sistema de Vigilância Alimentar e Nutricional. Brasília: MS; 2011.

14. World Health Organization (WHO). Child growth standards: length/height-for-age, weight-for-age, weight-for -length, weight-forheight 2006. [acessado 2013 abr 15]. Disponível em: http://www.who.int/childgrowth/standards/technical_report/en/

15. R Core Team. R: A language and environment for statistical computing. R Foundation for Statistical Computing [acessado 2014 nov 15]. Disponível em: http:// www.R-project.org/ 
16. Ferreira DC, Santos MA, Ravazzani EDA. Avaliação do perfil nutricional de crianças de um CMEI da região de Curitiba-PR. Cadernos da Escola de Saúde 2011; 2(6):90-99.

17. Vliet JS, Gustafsson PA, Duchen K, Nelson N. Social inequality and age-specific gender differences in overweight and perception of overweight among Swedish children and adolescents: a cross-sectional study. $B M C$ Public Health 2015; 15:628.

Artigo apresentado em 25/05/2015

Aprovado em 22/11/2015

Versão final apresentada em 24/11/2015 